Beyond spelling: The writing skills of students with dyslexia in higher education

Tops, W., Callens, C., Van Cauwenberghe, E., Adriaens, J., \& Brysbaert, M.

Ghent University, Belgium

Corresponding Author Address:

Wim Tops

Department of Experimental Psychology

Ghent University

Henri Dunantlaan 2

B-9000 Gent

Belgium

Tel. +3292649431

wim.tops@ugent.be 


\begin{abstract}
To have a clearer idea of the problems students with dyslexia may face during their studies, we compared writings of 100 students with dyslexia and 100 age matched control students in higher education. The aim of this study was to compare the writing of young adults with dyslexia and young adults without dyslexia. The study was carried out in Belgium with writers of Dutch. First, we studied the number and type of spelling errors, the quality of the texts produced, the use of words, and the handwriting, both in a précis writing task (writing a summary of an informative text) and in a dictation task (sentence writing). Our results showed medium to large effect sizes for spelling errors: $d=.93$ for morphosyntactic spelling errors, $d$ $=.55$ for memory-related spelling errors, and a medium effect size for punctuation and capitalization errors, $d=.40$. Second, experts who were blind to the aims of the study were asked to judge the quality of the writing of both groups based on transcriptions that were free from spelling errors. The quality of the texts produced was judged lower for students with dyslexia than for the controls, $d=.61$ for text structure and $d=.56$ for agreeability, even though the number and types of words used by both groups were very much the same. There was no significant difference in the quality of the handwriting, $d=.15$. Given that remedial teaching has been shown to be effective for essay-writing skills, educational support along these lines may be helpful for students with dyslexia.
\end{abstract}

Keywords: adult dyslexia - dictation - précis writing - spelling - writing 


\section{Beyond spelling: The writing skills of students with dyslexia in higher education}

A non-negligible percentage of students in mainstream higher education have learning difficulties (Hatcher, Snowling, \& Griffiths, 2002). Because they are expected to reach the same objectives as their peers, it is important for them to know how they can optimize their performance and for education authorities to have information about which types of support are helpful and justifiable. For instance, students with dyslexia are known to have spelling problems in addition to reading difficulties (Callens, Tops, \& Brysbaert, in press; Swanson \& Hsieh, 2009). Because written reports form the basis of many student assessments and evaluations (Farmer, Riddick, \& Sterling, 2002) and because poor spelling is likely to influence marking, it is reasonable to provide these students with text-processing software that has a built-in spelling checker (Desoete, Brysbaert, Tops, Callens, De Lange, \& Van Hees, 2010; Goldberg, Russell, \& Cook, 2003). At the same time, it would be good to know whether the quality of spelling-corrected essays and summaries is equivalent for students with dyslexia as for controls, or whether this is another challenge students with dyslexia should take into account.

Hayes and Flower (1980) proposed an influential cognitive model of skilled adult writing, analysing different components of the writing process and their interaction. In this model three linguistic processes, namely planning, translating, and reviewing, interact with one another. Writing is also influenced by non-linguistic processes such as long-termmemory and task environment, containing different aspects outside the writer, like the assignment, the goals, knowledge about the topic and the audience, and writing strategies. A final component of the model of Hayes et al. (1980) is monitoring, which is a metacognitive control processor or an executive function. In a modification of Hayes and Flower's model, Berninger, Fuller, and Withaker (1996) added working memory to account for beginning and developing writing. There is evidence that in people with learning disabilities, some of these 
processes might be impaired. Translation of ideas into language and its orthographic representation invokes (among many other processes) phonological encoding. According to Vellutino, Fletcher, Snowling, and Scanlon (2004), impaired phonological coding is the core deficit in dyslexia. Besides the phonological deficit hypothesis, other theories claiming that impaired working memory or inefficient executive control contribute to the reading and spelling difficulties from people with dyslexia, gain importance (Van der Sluis, de Jong, \& van der Leij, 2007). Executive functions are a number of skills that help us to adapt to new and changing situations. These functions allow acting in an effective and adequate way. Within this theory, weak working memory capacities evoke reading and spelling deficits in individuals with dyslexia. This could also offer an explanation for the specific writing problems of these individuals and explain why their writings are different from those of nondyslexic peers.

A review of the literature returned surprisingly few empirical studies addressing this question, nearly all of them dealing with the English language. Results from English writing research cannot automatically be generalized to other languages, such as Dutch, for two reasons. First, languages vary in the transparency of their correspondence rules. There are two types of correspondence rules: from letters to sounds and from sounds to letters. The latter are especially involved in correct spelling (Stone, Vanhoy, \& Van Orden, 1997). Alphabetical languages differ in the degree of transparency of these correspondence rules (Van den Bosch, Content, Daelemans, \& de Gelder, 1994) with English being more opaque, and Dutch more towards the transparent end of the continuum. Second, generalization of English findings to non-English-speaking countries may be hindered by the differences in the organization of the education system. British-inspired education is based on ability-based selection and a masterapprentice model. In many other countries, such as Belgium, there are no hard entrance criteria for higher education. Selection takes place as part of the curriculum (Callens et al. , in 
press). Ability-based admission criteria are likely to have implications for writing skills of the students since writing constitutes an important part of the SAT-scores in the US and of the Alevels in the UK.

In addition, most publications reported clinical practices, based on teachers' evaluations and self reports from students with dyslexia, rather than on a comparison of a group of students with dyslexia and a control group. Few studies included sample sizes large enough to have sufficient power, and hardly any used blind scoring.

Osborne (1999) reported a pilot study investigating the performance of higher education students with dyslexia in written assessments. She examined the marks of students with dyslexia in two types of written assessment: coursework and exams. The students known to have dyslexia had special provisions in the exams: they were allowed extra time and, if desired, could make use of a word processor. Despite these provisions, analysis of the marks showed that the students with dyslexia obtained lower scores than the control group, particularly on the exams.

Farmer, Riddick, and Sterling (2002) compared the writing skills of four higher education students with dyslexia with four age matched control students without learning problems. The students received various writing tasks including a free narrative task. The authors reported that the students with dyslexia scored worse than the control students with regard to multisyllabic words, the number of spelling and lexical errors, as well as the number of grammatical errors involving verbs and function words. However, they found no differences in the average number of written words, the number of text construction errors ${ }^{1}$, or punctuation errors. As for vocabulary and semantics, Farmer et al. reported that students with dyslexia were more prone to using simple and familiar words and sentences.

\footnotetext{
${ }^{1}$ These included unnecessary words, repetition, omission of proper nouns and sentence structure.
} 
Hatcher, Snowling, and Griffiths (2002) compared the performance of university students with dyslexia to that of an age matched control group without learning problems. To measure writing performance, they asked participants to write a summary of a text. The assessment included measures of content, structure, and readability. The authors concluded that the contents of the summaries written by students with dyslexia were poorer, mainly because of a lack of structure in the text. In contrast, Connelly, Campbell, MacLean, and Barnes (2006), after a comparison of 21 university students with dyslexia with 20 controls, argued that the students with dyslexia only differed in lower order transcription skills such as spelling and handwriting fluency. No differences were found in higher order variables, such as sentence length and lexical diversity. Although the holistic essay quality score of the group with dyslexia was significantly below that of the control, the authors argued that in absolute terms the divergence was not large and could be explained on the basis of differences in spelling abilities (the spelling errors were retained in the typed protocols and were judged by a single marker). On the basis of their findings, Connelly et al. (2006) concluded that students with dyslexia had poorer spelling and punctuation skills than their chronological-age controls, but were not lacking in ideas, organization, sentence construction, or grammar.

More studies on writing and composition skills have been done with primary and secondary school children. However, writing is a complex process that is subject to developmental changes. Berninger, Fuller, and Whitaker (1996) modified Hayes et al.'s (1980) model of skilled adult writing to explain beginning and developing writing. Berninger et al. (1996) argued that the processes involved in skilled adult writing cannot fully account for beginning and developing writing. Planning, translating, and revising are not fully operative in young writers. These skills originate as writing develops. In beginning writers, these processes are still developing, each at their own rate (Berninger et al., 1996, p. 198) 
Therefore, generalisation of findings in the writing of young children to adolescents and adults should not be taken for granted.

Nevertheless, we want to present the results of one study that was done with secondary school children, namely that of Therrien, Hughes, Kapelski, and Mokhtari (2009) because of its original design and its proof of effect of writing intervention. Therrien et al. (2009) compared essay-writing skills in 13 -year olds $\left(7^{\text {th }}\right.$ and $8^{\text {th }}$ grade) with and without reading and writing difficulties. They examined the effectiveness of a 6-step special education program teaching the students how to analyze the essay prompt, to outline and write a response, and to review the answer. Therrien et al. reported a strong effect of the training program on the writing strategies taught, $d=1.7$ but also a significant effect, $d=.7$, on a general essay measure consisting of six analytical traits: ideas and content, organization, voice, word choice, sentence fluency, and conventions. At the same time, the students with reading and writing difficulties after training still performed below the level of the control group on the general essay measure. However, the study involved major limitations. No IQ-measure was taken of the participants, the control group consisted of average achievers nominated by their teachers, and the essays were evaluated by two school psychology graduate students. Evaluation was blind but in all likelihood (this is not mentioned explicitly) happened on the basis of the original written forms.

All in all, there is a body of research suggesting that students with dyslexia may have poorer writing skills, which are not limited to the well-documented spelling problems. However, the evidence is limited, based exclusively on the English language, and obtained with a highly varied methodology. Furthermore, the conclusions are not shared by all researchers involved (see in particular Connelly et al., 2006). Farmer et al. (2002) offered a number of reasons as to why there may be so little research in the field of writing aptitude. A first possibility is that writing is a highly complex activity, of which only a small part can be 
examined in a single study. Because most studies tend to focus on reading and spelling difficulties and on poor phonological proficiencies, the higher-order writing skills of students with dyslexia receive little attention. A second reason may be that most researchers assume that students with dyslexia in higher education are not deficient at producing a well-written text, given that they have successfully completed a multitude of written exams to reach this level. Finally, it might be that writing skills have been overlooked because most adults with dyslexia no longer find writing problems their most pressing concern.

In the present study, we analyzed written reports of students with dyslexia in higher education to know whether they experience specific problems when they are faced with written assignments. This is done by comparing the output of students with dyslexia to that of an age and education matched control group. The results are based on two groups of 100 participants, so that we could reliably detect effect sizes as small as $d=.40$ (Cumming \& Finch, 2001). In clinical practice, effect sizes from $d=.50$ become practically relevant, because then we start to see a clear difference between the two population distributions.

The following research questions were addressed:

1. Do examiners who are blind to the nature of the study evaluate text summaries of students with dyslexia significantly differently from those of age and education matched students without learning problems, when the texts are typed and spelling errors are omitted?

2. If present, are such differences situated at the level of words, sentences, and/or text?

3. Does the handwriting of students with dyslexia differ from that of students without learning problems?

4. Is there a difference in writing quality of students with dyslexia on a dictation task as opposed to a text summary task? 


\section{Method}

\section{Participants}

We recruited 100 higher education students with dyslexia. The students were all in the first year of an academic or professional bachelor's program (34 university students and 66 students from non-university colleges). Recruitment happened with the help of a non-profit organization, Cursief, which is the main special education office for higher education in the city of Ghent, serving both the university and the other colleges. It provides assessment of learning disabilities and support to students having received such an assessment. All students were retested by us, to confirm the previously given clinical diagnosis.

In addition to the 100 students with dyslexia, 100 control students were recruited to participate in the study. This group was matched on age, gender, and current education level. Reading and spelling tests were administered to all control students to rule out reading problems. These consisted of a word-reading test (the Een Minuut Test; Brus \& Voeten, 1999), a non-word reading test (the Klepel; van den Bos, Spelberg, Scheepstra, \& de Vries, 1994) and a word dictation (De Pessemier \& Andries, 2009). None of the participants in the control group had other learning problems or a neurological disorder (such as autism, dyscalculia or ADHD).

All participants spoke Dutch as their first language and had normal or corrected vision. They took part in two tests sessions of three hours in return for a monetary reward. The main features of the two groups are shown in Table 1. The students with dyslexia did not differ from their peers in fluid IQ. However, the participants with dyslexia were significantly slower in word and pseudoword reading and they made significantly more errors in a word dictation. More details about the full range of tests administered can be found in Callens et al. (in press). 
Table 1:

General Information About the Student Groups With and Without Dyslexia

\begin{tabular}{lcc} 
Subject data & Dyslexia & No dyslexia \\
\hline Number & 100 & 100 \\
Male/Female & $46 / 54$ & $46 / 54$ \\
Mean age & $19 ; 4$ & $19 ; 11$ \\
University/Non-university & $66 / 34$ & $66 / 34$ \\
Fluid IQ (KAIT) & 105.4 & 106.8 \\
Number of words read in 1 min & 79 & $101 *$ \\
Number of pseudowords read in 1 min & 46 & $63 *$ \\
$\begin{array}{l}\text { Number of words correctly spelled } \\
\text { in a word dictation task (max = 30) }\end{array}$ & 17 & $24 *$
\end{tabular}

Note. Fluid IQ: mean $=100, \mathrm{SD}=15 ; * d>1.5, p<.01$

\section{Instruments}

We took inspiration from Hatcher et al. (2002), who asked participants to silently read a text and write a summary. In the present study, we asked the students to write a summary of an informational text.The skills assessed by this free-writing task are central to higher education and form a nice compromise between dictation and essay-writing (the outcome of which may be quite variable). All students were asked to silently read the text 'How Dangerous is a Tick Bite' from the Protocol Dyslexia Voortgezet Onderwijs [Dyslexia Protocol Higher Education], a Dutch text used for the assessment of dyslexia (Kleijnen et al., 2008). This is a rather simple but informative text explaining the risks of a tick bite, Lyme disease, how to deal with a tick bite, and how to prevent this. The text was originally written to measure passage reading fluency in 13-year old pupils at the start of secondary education. 
We preferred a text that was too easy but still appealing in content, so that it could easily be understood by all participants. In this way we avoided the problem that differences in writing were due to differences in text understanding.

The students were asked to write a summary of the text on a specially designed answer sheet (lined). They were not allowed to use scrap paper or to consult the text again. There were no length limits, but the students were encouraged to make the summary as complete and clear as possible. Since it has been shown that students with dyslexia need more time to read their texts and that their handwriting is slower than that of non-dyslexic peers (Callens et al., in press; Hatcher et al., 2002; Swanson \& Hsieh, 2009), there was no time limit.

\section{Procedure}

To process the summaries, the handwritten texts were typed in a standard form using a conventional text processor. This was done by five students from Ghent University (Belgium) who were majoring in Speech Therapy. The text was typed without spelling errors (these were recorded and classified separately; see below), but retained the original syntax, punctuation, and capitalization of letters. The essays were typed according to these criteria to prevent the raters from being influenced by the handwriting or spelling errors. Previous research has shown that handwriting characteristics may influence the evaluation of the written product (Defrancq \& Van Laecke, 2009). The quality of the handwriting was judged separately on the basis of the original written texts by raters asked not to take into account the quality of the text.

To judge the quality of the texts and the handwriting, we contacted teachers from the final years of secondary education, who were not informed about the fact that the study investigated dyslexia. Farmer et al. (2002) argued that high school language teachers have 
homogeneous experiences and expectations, resulting in more reliable and comparable scores.

Table 2 gives a summary of the profile of the teachers in terms of age and experience.

Table 2:

Teachers' Age and Experience

Teachers Age Experience

\begin{tabular}{lllll} 
& Mean & $(S D)$ & Mean & $(S D)$ \\
\hline Teachers readability & $46 ; 05$ year & $(13 ; 68)$ & $18 ; 10$ year & $(9 ; 93)$
\end{tabular}

Teachers handwriting $44 ; 08$ year $\quad(9 ; 62) \quad 19 ; 05$ year $\quad(14 ; 29)$

Readability of the texts was judged independently by ten language teachers. The teachers were given clear instructions on the assessment criteria. These were based on previous research (Defrancq \& Van Laecke, 2009; Farmer et al., 2002). One of the criteria, for instance, was that a good summary requires little cognitive effort to read; it is a text that can be read quickly and without having to be reread (Defrancq \& Van Laecke, 2009). For each criterion, a five-point Likert scale was used. Prior to the assessment process, the teachers were given five divergent writing products to judge, so that they could ask for clarifications and had a good idea of what we expected from them.

Handwriting was rated by ten different language and science teachers, all teaching in the final year of secondary education. Handwriting characteristics included the visual aspects of a hand-written text, such as consistency in letter size and slant, neatness, transition between letters, consistency between lines, spacing between words, cross-outs, and so on. These are qualities which have nothing to do with the language or content of the text but nevertheless have a great influence on the readability of the product (Defrancq \& Van Laecke, 2009). The teachers were asked to assign a rating to each piece of writing via a five-point Likert scale, ranging from very sloppy (1) to very good (5). 
The third component of our analysis involved the spelling errors. There is a welldocumented difference in the description of spelling and spelling errors between Dutch and English. In Dutch the individual graphemes matter, whereas in English in particular the distinction between onset and rhyme is important due to the many inconsistencies in the rhymes (Martensen, Maris, \& Dijkstra, 2000). For this reason and because it is common practice in the Dutch-speaking psycholinguistic research community, we preferred to use the Verkort Utrechts Classificatiesysteem voor Spelfouten [Short Classification System of Spelling Errors] (Castelijns, Hamers, \& De Koning, 1994; Kleijnen, 1992). This system classifies errors into three main categories: phonological, morphosyntactic, or memory-based spelling errors. Phonological errors are errors in which the simple phoneme-to-grapheme correspondence rules are violated. The student spells the word differently than it is pronounced. The most frequent examples of errors in this category are letter additions (e.g., *yorghurt instead of yoghurt), letter omissions (e.g., *delberate instead of deliberate), and letter substitutions (e.g., * drakness instead of darkness). Morphosyntactic spelling errors are errors in which specific morphosyntactic rules regarding the Dutch word spelling are violated. In Dutch, the morphosyntactic rules that have the most influence on spelling concern the rules to write (homophonic) verb forms, and conventions about how to write short and long vowels in open and closed syllables (e.g., *boomen [trees] instead of bomen [trees]). Memory-based spelling errors involve exception words that must be memorized because they lack specific spelling rules and they do not respect the simple phoneme-to-grapheme correspondence rules (e.g., *autun instead of autumn). They are often loan words from other languages (French, English, and German). For a more detailed overview of spelling errors in Dutch, we refer to Vanderswalmen, Vrijders, and Desoete (2010).

In addition to the above three categories, we also scored non-spelling-related errors, such as punctuation and capitalization errors, word omissions, additions, word order errors, 
anaphoric errors, semantic errors, and neologisms. Punctuation errors involve the omission or wrong use of punctuation marks. Because punctuation marks usually have implications for the capitalization of letters (e.g., in Dutch the first letter of a word must be capitalized after a full stop, but not after a colon or a semicolon), we grouped capitalization errors with punctuation errors. A word omission is a word that is not written down (but dictated or needed in a sentence context) and is not replaced by another word or phrase (e.g., *The father gave children a present instead of The father gave his children a present). When a word or phrase is added but is not dictated or not appropriate in a sentence context, it is called a word addition. The use of improper word order whereby syntactic rules are violated is called a word order error. Next, anaphoric errors involve the erroneous use of reference words such as *The cow, he gives milk instead of The cow, she gives milk. A semantic error occurs when a word or statement is syntactically valid, but is not appropriate at the lexical semantic level (e.g., *tennis bat instead of tennis racquet). Making up a new word, or adding a redundant or prefix or suffix to an existing word is called a neologism (e.g., *subliminible instead of subliminal).

The spelling and the non-spelling errors of the summary task were compared to those made in a sentence dictation task (Algemene Toets- Gevorderde Spelling van het Nederlands or AT-GSN [General Assessment of Advanced Dutch Spelling]; Ghesquière, 1998). The sentence dictation task was a high-level spelling test, developed especially for students entering higher education (in particular, at the University of Leuven, Belgium). It was taken by each student individually, according to the instructions in the handbook. Every sentence was first read out in its entirety and then broken down into sections and repeated in a uniform manner. No additional information was given by the examiner with regard to punctuation and capital letters. For the error analysis, the same classification system was used as for the text summary task. 


\section{Results}

The results are expressed as effect sizes, to facilitate the interpretation. The signs of the dvalues are adjusted so that a positive sign represents a better result by the students without learning problems. In order to investigate significance of the differences, Multivariate Analyses of Variance (MANOVA) were run.

Table 3 summarizes the group differences on the text summary task. We only found a significant difference for words longer than six letters, $F(23,157)=5.81, p<.05$. Thus students with dyslexia used fewer long words than age matched students without learning problems. Even then the difference in percentages was very small, $17 \%$ vs. $18 \% ; d=.36$.

Table 3:

Differences between Students With and Without Dyslexia on the Writing of Words, Sentences and Texts in the Text Summary Task

Categories

Dyslexia No dyslexia

\begin{tabular}{|c|c|c|c|c|c|c|}
\hline & M & $(S D)$ & M & $(S D)$ & $F$ & $d$ \\
\hline Number of syllables in a word & 1.5 & $(0.10)$ & 1.52 & $(0.09)$ & 2.09 & 0.21 \\
\hline Number of words & 141.32 & $(50.72)$ & 146.39 & $(49.04)$ & 0.47 & 0.10 \\
\hline Number of unique words & 90.7 & $(26.08)$ & 93.93 & $(25.21)$ & 0.72 & 0.13 \\
\hline Percentage high-frequency words ${ }^{1}$ & 78.88 & $(3.30)$ & 78.71 & $(3.26)$ & 0.11 & 0.05 \\
\hline Type token ratio $^{2}$ & 0.66 & $(0.07)$ & 0.66 & $(0.07)$ & 0.07 & 0.04 \\
\hline Proportion words of $>6$ characters & 0.17 & $(0.04)$ & 0.18 & $(0.03)$ & $5.81 *$ & 0.36 \\
\hline Number of sentences & 9.86 & $(3.43)$ & 9.9 & $(3.18)$ & 0.01 & 0.01 \\
\hline \multicolumn{7}{|l|}{ Mean number of words per } \\
\hline sentence & 14.57 & $(3.12)$ & 15.08 & $(3.12)$ & 1.24 & 0.17 \\
\hline
\end{tabular}

Note. ${ }^{1}$ By high frequency words, we mean words corresponding to the 500 most frequent words in a Dutch news paper corpus (available at: www.inl.nl/nl/corpora/27/miljoen/woorden/krantencorpus/1995), which 
together account for $77 \%$ of the words in that corpus. This metric was available in the text analysis software we used (available at: http://lt3.hogent.be/en/tools/classical-readability-formulas). ${ }^{2}$ The Type Token ratio is the ratio of different lexical items to the total number of lexical items, and is a measure of lexical diversity. $* p<.05$

Table 4 gives an overview of the evaluations of the summaries by the teachers. Several points are noteworthy. First, the general assessment of the structure and content of the summaries differed significantly between the two groups, $F(23,157)=6.76, p<.01$. The summaries of the group with dyslexia received a lower general score than those of the control group. The effect was small, however $(d=.39)$, and mainly due to differences in the structure and the agreeability of the essays, $F(23,157)=16.66, p<.01$ and $F(23,157)=14.39, p<.01$ respectively. The teachers thought that the essays of the students with dyslexia were more poorly structured than those of the age matched students without dyslexia. The difference was of a medium effect size, $d=.61$. The work of the students with dyslexia was also found to be less enjoyable to read than that of the age-matched students without learning problems. This also had a medium effect size, $d=.56$. The remaining factors (conciseness, vocabulary, and sentence structure) did not differ significantly between the two groups. Finally, the quality of the handwriting did not differ significantly either. In other words, the teachers did not see it as different or less neat.

Table 4:

Assessment of Content, Structure and Handwriting by the Panel of Teachers for the Text Summary Task

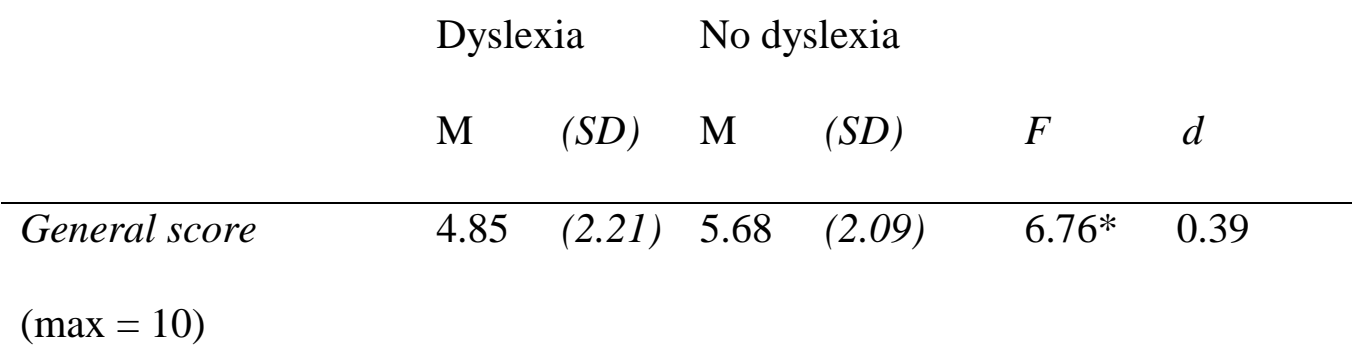

5-point Likert scales

$\begin{array}{lllllll}\text { Conciseness } & 0.81 & (0.79) & 0.97 & (0.80) & 1.69 & 0.19\end{array}$




\begin{tabular}{|c|c|c|c|c|c|c|}
\hline Structure & 1.62 & $(1.25)$ & 2.37 & $(1.22)$ & $16.66^{*}$ & 0.61 \\
\hline Agreeability & 1.55 & $(1.27)$ & 2.28 & $(1.32)$ & $14.39 *$ & 0.56 \\
\hline Vocabulary & 1.82 & $(0.69)$ & 1.99 & $(0.63)$ & 2.81 & 0.25 \\
\hline Sentence structure & 2.38 & $(1.22)$ & 2.34 & $(1.17)$ & 0.05 & -0.03 \\
\hline Handwriting & 2.63 & $(1.13)$ & 2.79 & $(1.05)$ & 1.00 & 0.15 \\
\hline
\end{tabular}

Table 5 gives the results of the error classification for the text summary task and the sentence dictation task. The students with dyslexia made significantly more spelling, and punctuation and capitalization errors than the control students both in the text summary task and in the sentence dictation task. Effect sizes for phonological errors, morphosyntactic errors, and punctuation and capitalization errors were medium $(.30<d \leq .60)$ for the summary task, but were almost twice as large for the sentence dictation task $(.60<d \leq 1.06)$. For the memory-related errors the effect size for text summary $(d=.36)$ was almost three times lower than for sentence dictation $(d=1.05)$. Not all non-spelling errors were present in both tasks because of the specificity of the assignment. The students with dyslexia omitted more words in the dictation task than their peers without learning disabilities, $F(11,188)=5.12, p=.030$. However, the effect size was small $(d=.16)$. In the text summary, the students with dyslexia made more semantic errors than the control group without dyslexia, $F(11,188)=2.45, p=$ .030. Again, the effect size was small $(d=.23)$. No important differences were found in the other error categories. 
Table 5:

Number and Type of Errors in the Text Summary Writing Task and the Sentence Dictation

\begin{tabular}{|c|c|c|c|c|c|c|c|}
\hline \multirow[t]{2}{*}{ Error type } & \multirow[t]{2}{*}{ Task } & \multicolumn{2}{|c|}{ Dyslexia } & \multicolumn{2}{|c|}{ No dyslexia } & \multirow[b]{2}{*}{$F$} & \multirow[b]{2}{*}{$d$} \\
\hline & & M & $(S D)$ & M & $(S D)$ & & \\
\hline \multirow[t]{2}{*}{ Phonological errors } & Summary & 1.21 & $(1.93)$ & 0.35 & $(0.61)$ & $51.29 * *$ & 0.43 \\
\hline & Dictation & 7.42 & $(6.05)$ & 2.73 & $(2.51)$ & $17.67 * *$ & 0.72 \\
\hline \multirow[t]{2}{*}{ Morphosyntactic errors } & Summary & 3.34 & $(2.37)$ & 1.55 & $(1.78)$ & $112.89 * *$ & 0.60 \\
\hline & Dictation & 25.13 & $(9.54)$ & 12.95 & $(6.36)$ & $36.41 * *$ & 1.06 \\
\hline \multirow[t]{2}{*}{ Memory-related errors } & Summary & 0.97 & $(1.18)$ & 0.44 & $(0.88)$ & $109.20 * *$ & 0.36 \\
\hline & Dictation & 15.50 & $(8.43)$ & 5.92 & $(3.60)$ & $13.02 * *$ & 1.05 \\
\hline \multirow{2}{*}{$\begin{array}{l}\text { Punctuation and } \\
\text { capitalization errors }\end{array}$} & Summary & 2.88 & $(2.71)$ & 1.79 & $(2.30)$ & $9.39 * *$ & 0.31 \\
\hline & Dictation & 4.39 & $(2.80)$ & 2.50 & $(1.42)$ & $32.84 * *$ & 0.60 \\
\hline \multirow[t]{2}{*}{ Word omission errors } & Summary & 0.69 & $(2.01)$ & 0.38 & $(0.69)$ & 2.13 & 0.15 \\
\hline & Dictation & 0.60 & $(1.09)$ & 0.36 & $(1.08)$ & $2.45^{*}$ & 0.16 \\
\hline \multirow[t]{2}{*}{ Word addition errors } & Summary & 0.27 & $(0.69)$ & 0.16 & $(0.53)$ & 1.59 & 0.13 \\
\hline & Dictation & 0.09 & $(0.35)$ & 0.03 & $(0.17)$ & 2.36 & 0.15 \\
\hline \multirow[t]{2}{*}{ Word order errors } & Summary & 0.26 & $(0.82)$ & 0.08 & $(0.27)$ & 4.30 & 0.21 \\
\hline & Dictation & -- & -- & -- & -- & -- & -- \\
\hline \multirow[t]{2}{*}{ Anaphoric error } & Summary & 0.09 & $(0.32)$ & 0.11 & $(0.31)$ & 0.20 & -0.04 \\
\hline & Dictation & -- & -- & -- & -- & -- & -- \\
\hline \multirow[t]{2}{*}{ Semantic error } & Summary & 0.23 & $(0.49)$ & 0.10 & $(0.30)$ & $5.12 *$ & 0.23 \\
\hline & Dictation & 0.47 & $(0.75)$ & 0.42 & $(1.03)$ & 0.16 & 0.04 \\
\hline \multirow[t]{2}{*}{ Neologism } & Summary & 0.20 & $(0.45)$ & 0.10 & $(0.33)$ & 3.19 & 0.18 \\
\hline & Dictation & -- & -- & -- & -- & -- & -- \\
\hline
\end{tabular}

Note. $* p<.05 ; * * p<.01$ 
To examine whether the types of errors differed between students with and without dyslexia and between the two tasks, we were confronted with the fact that the response categories (correct, phonological error, memory-related error, and morphosyntactic error) were not independent, making the use of analysis of variance questionable. ${ }^{2}$ A solution to this problem is to work with a generalized linear mixed model with a poisson link function (see the SPSS help files for more information). Although the dependent variable is categorical, a poisson distribution is a good representation of the data (Agrestie, 2002), given that there was no limit to the number of errors (especially in the text summary).

The fixed effects in our model were group (dyslexic, control), task (sentence dictation, text summary), and type of error (memory-related, morhposyntactic and phonologycal errors). Task and type of error were repeated factors within participants . The random slopes were significant for type of error $\left(\chi_{(7)}^{2}=81.733, p<.001\right)$, for task $\left(\chi_{(4)}^{2}=86.242, p<.001\right)$, and for their interaction $\left(\chi_{(11)}^{2}=28.604, p<.001\right)$ and so were added to the model (in addition to the random intercept per participant), in order to minimize the within-subject correlations of the model's error term.

There was a main effect of group, $\chi_{(1)}^{2}=111.91, p<.001$. In the dyslexic group there were 2.46 times more errors than in the control group. There was a main effect of task $\left(\chi_{(1)}^{2}=\right.$ 1104.23, $p<0.001)$. As could be expected from the fact that the dictation task comprised many words with difficult spellings, which could be avoided in the text summary task, the number of errors was about 10 times higher in the sentence dictation task than in the text summary task. There was also a main effect of error type, $\chi_{(2)}^{2}=646.88, p<.001$. There were 2.79 times more morphosyntactic errors than memory-related errors, $\chi_{(1)}^{2}=335.25, p<.001$,

\footnotetext{
${ }^{2}$ The authors thank an anonymous reviewer for pointing them to this problem. They thank Michael Stevens for proposing and running the alternative analysis. Readers may additionally be interested to know that the conclusions on the basis of the alternative analysis did not differ from those of the original ANOVA we ran.
} 
and 1.70 times more memory-related errors than phonological errors, $\chi_{(1)}^{2}=40.92, p<0.001$. The number of morphosyntactic errors was 4.75 times higher than the number of phonological errors, $\chi_{(1)}^{2}=464.86, p<0.001$.

The interaction between task and error type was also significant $\left(\chi_{(2)}^{2}=42.03, p<\right.$ 0.001). In the sentence dictation morphosyntactic errors were more frequent than memory related errors $\left(\chi_{(1)}^{2}=485.89, p<.001\right)$ which in turn were more frequent than phonological errors, $\chi_{(1)}^{2}=284.88, p<.001$. In the text summary morphosyntactic errors were also more frequent than memory related errors $\left(\chi_{(1)}^{2}=167.06, p<.001\right)$, but memory related errors were not more frequent than phonological errors, $\chi_{(1)}^{2}=1.49, p=.222$.

Finally, the interaction between group and error type reached significance as well, $\chi^{2}{ }_{(2)}$ $=6.41, p=0.041$. Further analysis revealed that this was due to the fact that the difference between phonological and morphosyntactic errors was larger in the control group than in the dyslexic group (5.61 vs. 4.02 times, $\left.\chi_{(1)}^{2}=5.30, p=0.021\right)$. The difference between phonological and memory-related errors and between morphosyntactic and memory-related errors was similar in both groups, $\chi_{(1)}^{2}=0.82, p=.363$, and $\chi_{(1)}^{2}=2.63, p=.104$. So, the students with dyslexia made relatively less morphosyntactic errors and more phonological errors. The error profiles of students with and without dyslexia are shown in Figure 1 (see also Table 5 for the data). 


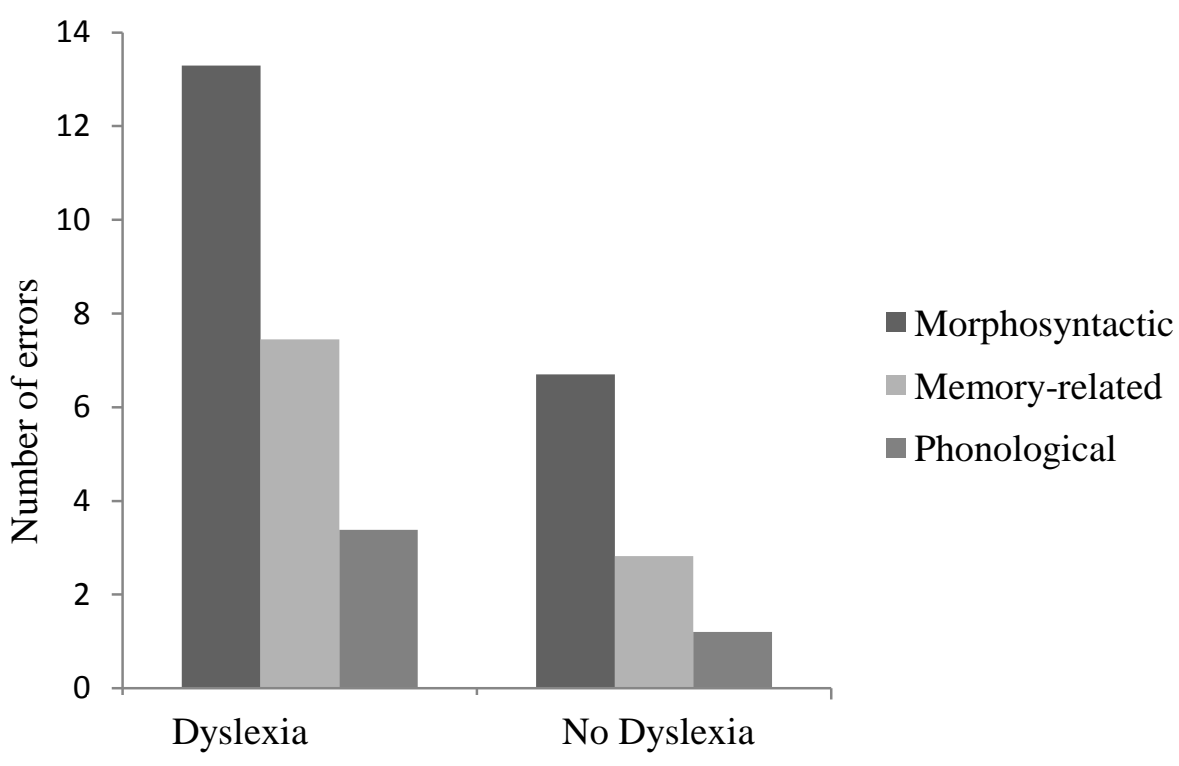

Figure 1. Interaction between group and error type.

\section{Discussion}

The aim of this study was to discover if the writing skills of students with dyslexia differ from those of peers without learning problems. This was done by asking the participants to write a sentence dictation and the summary of a text just read silently. Several variables were measured:

1. Spelling, punctuation and capitalization errors in both tasks.

2. Words and syntax used in the text summary task.

3. Evaluation of the quality of the summaries when the spelling errors were removed.

4. Attractiveness of the handwriting.

Despite the proficiency of our research groups (students in higher education), the spelling errors were much more frequent in the students with dyslexia than in the controls. As a matter of fact, the various spelling tests made the largest distinctions between both groups (with effect sizes of more than $d=2$ ), even more than word or nonword reading tests (Callens 
et al., in press). The extra mistakes were present for all three categories of word spelling errors (morphosyntactic, memory-related, and phonological), making that the error profiles look very similar for students with dyslexia and the controls (Figure 1). In relative (but not absolute) terms the students with dyslexia made slightly less morphosyntactic errors, possibly as the result of the remedial teaching they received in primary and secondary education (in which the morphological spelling rules are stressed). In addition, we observed that individuals with dyslexia find it difficult to use proper punctuation and capitalization. They also made more word order errors. Similar results were reported in an article by Gregg, Coleman, Davis, and Chalk (2007), but not in the study of Farmer et al. (2002). In the latter case, it should be taken into account that the numbers of participants tested (four per group) only allowed the authors to observe very large effect sizes.

The only form-related parameter showing a significant difference in the text summary task was that of words longer than six letters. This is in line with the preliminary findings of Farmer et al. (2002), who found a difference between the scores of students with dyslexia and controls for multi-syllable words as well. Apparently, students with dyslexia spontaneously tend to avoid long words. Other parameters such as sentence length and average word count showed no significant differences. In general, both students with dyslexia and control students tended to avoid words with tricky spellings in spontaneous writing, given that the number of errors was much lower in the text summary task than in the sentence dictation task.

In addition to a quantitative analysis of the text summary products, we also had the writing samples evaluated by teachers blind to the nature of the study and on typed protocols without spelling errors. In line with the quantitative analysis, the teachers did not see differences in vocabulary or sentence structure, as displayed in Table 4. Still, the summaries of the students with dyslexia obtained lower marks than those of the control participants, $d=$ .40 , due to the overall structure of the text and the agreeability of the text. The essays of 
students with dyslexia were not as well structured and could not be read as fluently as those of the control group. This is likely to have implications for the evaluation of texts written by students with dyslexia as assigned work or for essay-type exams. Even if they master the topic to the same degree, their writings risk to be less highly regarded and therefore to get lower marks.

In this study we found evidence that the writing difficulties of young adults with dyslexia are not limited to the word and sentence level, but are present at the discourse level as well. This can be taken into account in remedial teaching programs for high functioning young adults with dyslexia. Given that essay-writing skills and discourse organisation can be taught to some extent (Therrien et al., 2009; see the introduction), this merits evaluation and consideration in intervention programs for dyslexia. Furthermore, it would be worthwhile to investigate higher level language organisation and oral expression in individuals with dyslexia. Based on the finding that students with dyslexia fail to write essays of as high quality as controls, it could be that dyslexia interferes with discourse organisation in spoken language as well.

With regard to handwriting, no significant differences were found between the two groups taking part in the study. In other words, the handwriting of students with dyslexia was not sloppier or less legible, according to the rating panel of teachers. This contrasts with previous studies showing a comorbidity of dyslexia with dysgraphia (Berninger et al., 2008; Iversen et al., 2005; Nicolson \& Fawcett, 2011). A possible explanation is that comorbidity tends to be less prevalent among the students starting higher education. Indeed, our group of students showed a profile of strengths and weaknesses very much in line with that of pure dyslexia (Callens et al., in press). 
All in all, our study confirms the persistent spelling problems of students with dyslexia and a significant difference in the coherence of the texts produced. We believe these are interesting aspects to focus on in educational support, given that spelling support software is easy to provide nowadays and that essay-writing skills can also be improved by a special education program.

\section{Acknowledgments}

This study was made possible by an Odysseus Grant awarded by the Government of Flanders to MB. The authors thank Valérie Van Hees and Charlotte De Lange from Cursief for their help in the study and the recruitment of participants. They also thank Joke Lauwers for her assistance in testing the participants, and Annemie Desoete and two anonymous reviewers for their helpful suggestions. 
References

Agrestie, A. (2002). Categorical Data Analysis (2nd edition). Hoboken, NJ: Wiley \& Sons, Inc.

Berninger, V.W., Fuller, F., \& Withaker, D. (1996). A process model of writing development across the life span. Educational Psychology Review, 8, 193-218._doi: 10.1007/BF01464073

Berninger, V., Nielsen, K. H., Abbott, R. D., Wijsman, E., \& Raskind, W. (2008). Writing problems in developmental dyslexia: Under-recognized and under-treated. Journal of School Psychology, 46, 1-21. doi: 10.1016/j.jsp.2006.11.008

Brus, B. T., \& Voeten, M. M. (1999). Eén-Minuut Test (EMT) [One Minute Test]. Lisse, The Netherlands: Swets.

Callens, M., Tops, W., \& Brysbaert, M. (2011). Cognitive profile of higher education students with an indication of dyslexia. Manuscript submitted for publication.

Castelijns, J., Hamers, J., \& De Koning, E. (1994). Verkort Utrechts Classificatiesyteem voor Spelfouten (V-UCS): Verantwoording en Handleiding [Shortened Utrecht classification system for spelling errors: Manual and validation]. Lisse, The Netherlands: Swets \& Zeitlinger.

Connelly, V., Campbell, S., MacLean, M., \& Barnes, J. (2006). Contribution of lower order skills to the written composition of college students with and without dyslexia. Developmental Neuropsychology, 29, 175-196.doi: 10.1207/s15326942dn2901_9 
Cumming, G., \& Finch, S. (2001). A primer on the understanding, use and calculation of confidence intervals based on central and noncentral distributions. Educational and Psychological Measurement, 61, 530-572._doi: 10.1177/0013164401614002

Defrancq, B., \& Van Laecke, G. (2009). Leesbaar schrijven [Clear writing]. Antwerpen, Belgium: Garant.

Depessemier, P., \& Andries, C. (2009). Gletschr, Dyslexie bij +16-jarigen [Gletschr, dyslexia in young adults]. Antwerp, Belgium: Garant.

Desoete, A., Brysbaert, M., Tops, W., Callens, M., De Lange, C., \& Van Hees, V. (2010). Studeren met Dyslexie [Dyslexia in higher education]. Ghent, Belgium: Department of Diversity and Gender Ghent University.

Farmer, M., Riddick, B., \& Sterling, C. (2002). Dyslexia and inclusion, assessment and support in higher education. Philadelphia, PA: Whurr Publishers.

Ghesquière, P. (1998). Algemene toets gevorderde spelling van het Nederlands (AT-GSN). Verantwoording en handleiding. Rapport van een specialisatiejaar: Onderzoek ATGSN-dictee. [General Assessment of Advanced Dutch Spelling. Manual and validation. Research report of a specialised course]. Leuven, Belgium: Catholic University of Leuven.

Goldberg, A., Russell, M., \& Cook, A. (2003). The effect of computers on student writing: A meta-analysis of studies from 1992 to 2002. Journal of Technology, Learning, and Assessment, 2. 4-51. Available at: https://ejournals.bc.edu/ojs/index.php/ jtla/article/viewFile/1661/1503. 
Gregg, N., Coleman, C., Davis, M., \& Chalk, J. C. (2007). Timed essay writing: Implications for high-stakes tests. Journal of Learning Disabilities, 40, 306-318. doi: $10.1177 / 00222194070400040201$

Hatcher, J., Snowling, M. J., \& Griffiths, Y. M. (2002). Cognitive assessment of dyslexic students in higher education. British Journal of Educational Psychology, 72, 119-133. doi: 10.1348/000709902158801

Hayes , J.R., \& Flower, L.S. (1980). Identifying the organisation of writing processes. In L.W. Gregg \& E.R. Sternberg (Eds.), Cognitive processes in writing (pp.3-30). Hillsdale, NY: Lawrence Erlbaum Associates, Inc.

Iversen, S., Berg, K., Ellertsen, B., \& TØnnessen, F. (2005). Motor coordination difficulties in a municipality group and in a clinical sample of poor readers. Dyslexia, 11, 217- 231. doi: $10.1002 /$ dys.297

Kleijnen, R. (1992). Hardnekkige spellingfouten: Een taalkundige analyse [Persevering spelling errors: A linguistic analysis]. Lisse, The Netherlands: Swets \& Zeitlinger.

Kleijnen, R., Bosman, A., De Jong, P., Henneman, K., Pasman, J., Paternotte, A. Ruijssenaars, A., et al. (2008). Diagnose en behandeling van dyslexie. Brochure van de Stichting Dyslexie Nederland. Geheel herziene versie [Diagnosis and treatment of dyslexia. Brochure of the Dutch Dyslexia Foundation. Revision]. Bilthoven, The Netherlands : Stichting Dyslexie Nederland.

Martensen, H., Maris, E., Dijkstra, T. (2000). When does inconsistency hurt? On the relation between phonological consistency effects and the reliability of sublexical units. Memory and Cognition, 28, 648-656. doi: 10.3758/BF03201254 
Nicolson, R. I., \& Fawcett, A. J. (2011). Dyslexia, dysgraphia, procedural learning and the cerebellum. Cortex, 47, 117-127. doi: 10.1016/j.cortex.2009.08.016

Osborne, P. (1999). Pilot study to investigate the performance of dyslexic students in written assessments. Innovations in Education and Training International, 36, 155-160. doi: $10.1080 / 1355800990360208$

Stone, G.O., Vanhoy, M., \& Van Orden, G.C. (1997). Perception is a two-way street: Feedforward and feedback phonology in visual word recognition. Journal of Memory and Language, 36, 337-359. doi: 10.1006/jmla.1996.2487

Swanson, L., \& Hsieh, C.-J. (2009). Reading disabilities in adults: A selective meta-analysis of the Literature. Review of Educational Research, 79, 1362-1390. doi: $10.3102 / 0034654309350931$

Therrien, W. J., Hughes, C., Kapelski, C., \& Mokhtari, K. (2009). Effectiveness of a testtaking strategy on achievement in essay tests for students with learning disabilities. Journal of Learning Disabilities, 42, 14-23. doi: 10.1177/0022219408326218

van den Bos, K., Spelberg, H., Scheepstra, A., \& de Vries, J. (1994). De Klepel. Een test voor de leesvaardigheid van pseudowoorden: Vorm A en $B$ [The Klepel. A reading test for pseudowords: Version A and B]. Nijmegen, The Netherlands: Berkhout.

Van den Bosch, A., Content, A., Daelemans, W., \& De Gelder, B. (1994). Measuring the complexity of Writing Systems. Journal of Quantitative Linguistics, 1, 178-188.

Van der Sluis, S., de Jong, P.F., \& van der Leij, A. (2007). Executive functioning in children, and its relations with reasoning, reading, and arithmetic. Intelligence, 35, 427-449. doi: 10.1016/j.intell.2006.09.001 
Vanderswalmen, R., Vrijders, J., \& Desoete, A. (2010). Metacognition and spelling performance in college students. In A. Efklides \& P. Misailidi (Eds.), Trends and prospects in metacognition research (pp. 367-394). New York, NY: Springer.

Vellutino, F., Fletcher, M., Snowling, M., \& Scanlon, D. (2004). Specific reading disability (dyslexia): What have we learned in the past four decades? Journal of child psychology and psychiatry, 45, 2-40. DOI: 10.1046/j.0021-9630.2003.00305.x 